\title{
New One-for-All Powder Coating System
}

$\mathrm{T}$ he innovative, polyester-based Alesta IP Gloss has been developed by DuPont CoatingSolutions for a broad range of industrial indoor and outdoor applications. The new glossy powder coating system features optimised application properties combined with high weathering and UV resistance as well as mechanical properties and a more flexible curing window. Alesta IP Gloss therefore fulfils the requirements in many areas of industrial powder coating. Powder coatings based on polyester are often the first choice when it comes to industrial coating of metal surfaces for indoor and outdoor use. The new powder coating system optimises functional, visual and economic advantages in the coating of all kinds of metals. The new product has been available since March 2012. A key feature of the new powder coating system is its enhanced application characteristics. Alesta IP Gloss exhibits excellent reactivity and very good flow. The coated, glossy surface provides excellent resistance against weathering and UV radiation. At the same time, it features good mechani-

Alesta IP Gloss also offers added value from an economic perspective. The high transfer efficiency results in reduced material consumption and high productivity. The more flexible curing window also helps to save energy. The versatile ap-

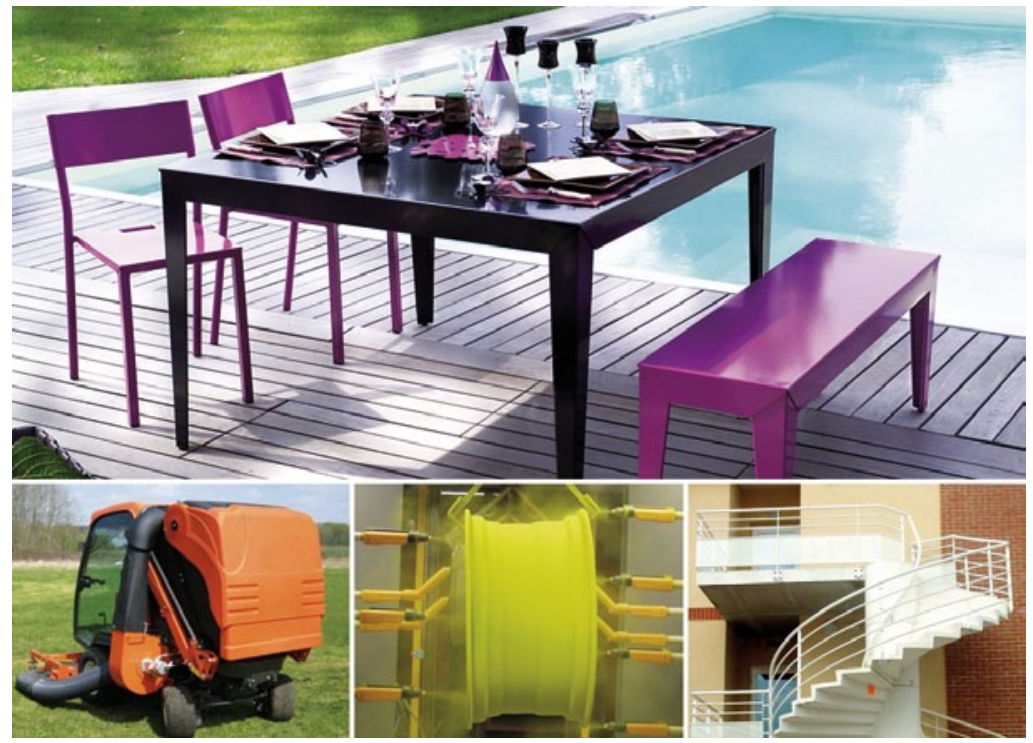
plication spectrum and the wide range of colours available mean that the new powder coating system can be used for protective and decorative coatings in practically all areas of industry. This versatility enables jobshop coaters in particular to reduce the number of powder coatings kept on stock, which leads to cost and space savings. To enable users to become familiar with these benefits in

cal properties and is highly flexible. Additionally, the new powder allows users to apply a higher layer thickness, for example between 150 and 250 microns, without even the slightest surface defect. practice, DuPont CoatingSolutions are offering a $1 \mathrm{~kg}$ can of Alesta IP Gloss free of charge for testing purposes. These can be ordered at www.ipgloss. de.

www.coatingsolutions.dupont.com

\section{Conductibility and Adhesiveness}

To guarantee the conductibility and adhesiveness of sensors for implants, e.g. for sensors made of polyamide 6 with a fiberglass piece and a head crafted out of galvanized nickelgold with a razor-thin layer (3-4 $\mu \mathrm{m}$ ) of bond gold, they are treated with plasma at Diener electronic in a class 8 cleanroom. The advantage of low-pressure plasmas consists in the fact that the treated items warm up only slightly. When activating synthetic materials made of polypropylene, the heating is hardly even measureable. The Tetra 50 system is equipped with a rotary drum that has holes in it. The parts are put into this

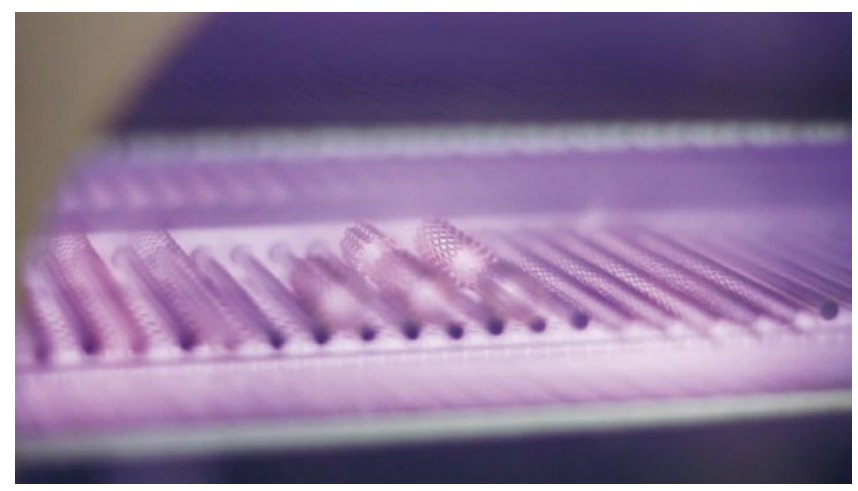

rotary drum as loose material. The rotational motion ensures that the parts are treated equally on all sides.

During plasma treatment energy is continuously fed to the material, its temperature increases, and it converts from a solid, to a liquid and then into a gas. If the energy continues to flow, the existing atomic shells break up and this results in charged particles, i.e. negatively charged electrons and positively charged ions. This mixture is called plasma. A vacuum and a pressure of around 0.4 mbar are also generated in the chamber of the machine. Then process gas enters and a voltage of $40 \mathrm{kHz}$ is generated on the electrodes. This is how the gas ionizes and a plasma results. The plasma removes organic layers from the sensors during treatment. These are attacked chemically by the oxygen used as process gas. The impurities partially evaporate from the vacuum and from the heat on the surface. The energy-rich particles transform the impurities into smaller, stable molecules and then they can be extracted. To guarantee complete traceability, all part-packages are labeled. Based on the information printed there, what charge is involved or when it was produced can be retraced - and the appropriate protocol can be assigned to it without any ambiguity.

Tel. +49 7458999 31-0, www.plasma.de 